\title{
Short Tear Break-Up Time and Seasonal Variation in
}

\section{Intra-Ocular Pressure}

\author{
Masahiko Ayaki*, Kazuno Negishi*, Kenya Yuki, Motoko Kawashima, Miki Uchino, and Kazuo \\ Tsubota, \\ 1 Otake Clinic Moon View Eye Center, Yamato, Japan (M.A.); ${ }^{2}$ Department of Ophthalmology, Keio University \\ School of Medicine, Tokyo, Japan (M.A., K.Y., M.K., M.U., K.T., K.N.) \\ *Correspondence: mayaki@olive.ocn.ne.jp; Tel.: +81-3-5363-3821; fwic7788@mb.infoweb.ne.jp; Tel: +81-3-5363- \\ 3821
}

M.A. and K.N. equally contributed to this work.

\begin{abstract}
:
Purpose: To evaluate seasonal variation in intra-ocular pressure (IOP) with and without short tear breakup time (SBUT, BUT $\leq 5 \mathrm{~s})$.

Methods: This study enrolled 176 patients who visited one of six eye clinics in Japan for IOP measurement at every season. The mean patient age was 67.9 years, including 79 males. We compared the seasonal variation in IOP (mean \pm SD) across spring (Mar-May), summer (Jun-Aug), fall (Sep-Nov), and winter (Dec-Feb).

Results: The IOP $(\mathrm{mmHg})$ in winter and summer, respectively, was $12.8 \pm 3.7$ and $12.8 \pm 3.1$ for nonglaucoma patients without SBUT $(\mathrm{n}=47, \mathrm{P}=0.964), 14.8 \pm 3.4$ and $13.3 \pm 3.4$ for non-glaucoma patients with SBUT $(\mathrm{n}=57, \mathrm{P}<0.001), 14.3 \pm 3.2$ and $14.1 \pm 3.4$ for glaucoma patients without $\operatorname{SBUT}(\mathrm{n}=36, \mathrm{P}=0.489)$, and $13.3 \pm 3.0$ and $11.6 \pm 2.9$ for glaucoma with SBUT $(n=36, P<0.001)$. Seasonal variation was largest across the seasons in the glaucoma with SBUT group, and the magnitude of seasonal variation correlated with
\end{abstract} $\operatorname{BUT}(\beta=0.228, \mathrm{P}=0.003)$.

Conclusions: Seasonal variation tended to be larger in patients with SBUT than those without SBUT.

Keywords: dry eye; tear break-up time; intra-ocular pressure; seasonality

\section{Introduction}

Glaucoma and dry eye disease (DED) are common geriatric diseases associated with poor quality of life and requiring ongoing daily topical medication $[1,2]$ The prevalence of glaucoma and DED in middle aged and elderly Japanese is $4 \%$ and $12-23 \%$, respectively, $[3,4]$ making both disorders a common presentation in daily 
ophthalmic practice. In addition, glaucoma and DED frequently co-exist in a single patient, and some of the glaucoma medications can affect the ocular surface as a side effect [5]. For this reason, glaucoma medication comes in a preservative-free, single dose unit, with reduced toxicity from chlorhexidine and SofziaR, and a decreased concentration of benzalkonium chloride (BAK), the most commonly used preservative in such agents. DED is also a multifactorial disease that predominantly affects women and the elderly, rheumatoid disease, and immunological disease, and that generally worsens in both prevalence and severity during cold and dry weather.[6-10] DED patients can have wide variety of symptoms and ocular surface signs, but short tear breakup time (BUT) has been introduced as a representative sign in most recent diagnostic criteria.[2]

There are many risk factors for glaucoma in terms of ocular hypertension and optic nerve damage,[11-14] with seasonality in intra-ocular pressure (IOP) well documented as well as higher IOP with hypertension and cold weather.[15-21] Clinically, it is unknown whether DED may alter IOP and therefore glaucoma, since both conditions worsen in winter, and both IOP and lacrimal secretion are under neural control.[22] Consequently, IOP control should be given special attention across all seasons. To investigate the clinical importance of IOP with and without DED, we compared seasonality between short and normal BUT cases with and without glaucoma. The obtained results should provide useful information for IOP control in these common diseases.

\section{Methods}

\subsection{Study Design, Ethical Approval, and Study Population}

This study was a multisite, hospital-based, cross-sectional, case-control study conducted from March 2015 to February 2017. The cases and control subjects were recruited from six clinical sites: Komoro Kosei General Hospital (Nagano, Japan), Shinseikai Toyama Hospital (Toyama, Japan), Tsukuba Central Hospital (Ibaraki, Japan), Jiyugaoka Ekimae Eye Clinic (Tokyo, Japan), Todoroki Eye Clinic (Tokyo, Japan), and TakahashiHisashi Eye Clinic (Akita, Japan).

This study was done on mainland Japan, where the latitude is 35.68 degrees North in the Tokyo area and day length varies by 4-6 hours over the year according to averages for 1981-2010 reported by the Japan Meteorological Agency. Japan has four distinct seasons during which the temperature, humidity, and daylight time markedly varies. Between 1981 and 2010, the average temperature and humidity in the Tokyo area ranged from $5.2^{\circ} \mathrm{C}$ and $52 \%$ in winter to $25.0^{\circ} \mathrm{C}$ and $77 \%$ in summer, in Akita from $0.1{ }^{\circ} \mathrm{C}$ and $73 \%$ in winter to $22.9^{\circ} \mathrm{C}$ and $79 \%$ in summer, in Toyama from $2.7^{\circ} \mathrm{C}$ and $79 \%$ in winter to $24.6^{\circ} \mathrm{C}$ and $78 \%$ in summer, and in Komoro from $-0.6^{\circ} \mathrm{C}$ and $78 \%$ in winter to $23.8^{\circ} \mathrm{C}$ and $74 \%$ in summer, respectively, as reported by the Japan Meteorological Agency. 
The respective institutional review boards and ethics committees of Shinseikai Toyama Hospital (Permit Number: 150503) and Komoro Kosei General Hospital (Permit Number: 2705) approved this study, which was conducted in accordance with the tenets of the 1995 Declaration of Helsinki (as revised in Edinburgh, 2000). Informed consent was obtained from all participants.

Participants were divided into four groups based on the presence of glaucoma and/or short BUT $(\leq 5$

seconds); non-glaucoma with normal BUT as control group, non-glaucoma with short BUT group, glaucoma without short BUT group, and glaucoma with short BUT group.

\subsection{Inclusion and exclusion criteria}

Patients were enrolled if they had their IOP measured during four consecutive seasons and received the same medication during the study period. Study participants visited our clinic for regular checkups for early cataract, eye fatigue, DED, and glaucoma. Patients were excluded from the study if they had visual impairment $(<20 / 25$ in either eye $)$ or were under 20 years of age. Patients were excluded from the study if they had history of past glaucoma surgery, or any ocular surgery within twelve months. We excluded patients from glaucoma cases if they have secondary glaucoma, uveitis, or steroid administration.

\subsection{Ophthalmological examinations}

IOP was measured in the morning session between 8 am and 12 midday, using a non-contact tonometer in all cases. The mean BUT and corneal staining scores (0-9 points) were based on the Japanese dry eye diagnostic criteria,[23] with BUT evaluated three times, and the mean value determined. Corneal fluorescein staining scores were evaluated in three areas and scored on a 0 - to 2-point scale (0: no damage to 2: damaged entirely); the scores were summed up to a maximum of 2 points in total. Ocular surface abnormality was defined as a BUT $\leq 5$ seconds and corneal staining scores $\geq 1$ point based on previous studies.[24,25]. The BUT and corneal fluorescein staining score were determined at the date of each patient's visit in year 2017 and classified as follows: spring from March to May, summer from June to August, fall from September to November, and winter from December to February.

All glaucoma cases were bilateral open angle glaucoma or normal tension glaucoma. For glaucoma diagnosis, we conducted a visual field test (Humphrey Visual Field Analyzer 30-2 standard program; Carl Zeiss, Jena, Germany), measuring the thickness of ganglion cell complexes using optical coherent tomography (OCT; 
RC3000R (Nidek, Gamagori, Japan) and Cirrus ${ }^{\circledR}$ HD-OCT (Carl Zeiss, Jena, Germany)), and then routine examinations were performed. As described previously [24], diagnostic criteria for glaucoma in the present study comprised glaucomatous visual field loss tested using the Glaucoma Hemifield Test, an ophthalmoscopic neurofiber layer defect, a cup/disc ratio $>0.6$, or elevated IOP $(>21 \mathrm{mmHg}$ ) requiring topical medication for more than 6 months. We confirmed no change in glaucoma medication during study period with chart review. Exclusion criteria were coexisting cataract with significant lens opacity disturbing the optical axis that accounted for subjective visual disturbance or decreased visual function, retinal pathology, retinal surgery, or photocoagulation affecting the visual field. Topical glaucoma medications were listed in Table S1.

\section{Statistical analysis}

The effects of each season on the IOP were compared as follows. Based on a preliminary study and previous investigations suggesting that IOP is highest in winter and lowest in summer, we identified the mean IOP for each season and then compared summer and winter using by t-test or Mann-Whitney U test with Bonferroni correction to determine if the differences between seasons were significant. Mean IOP and the magnitude of seasonality among the four study groups were also compared. Regression analysis was performed to explore which parameters most affect the magnitude of seasonal difference in IOP. Ophthalmological parameters and glaucoma medications were analyzed with multiple regression from the first analysis since most cases were prescribed with PG as a baseline and beta blocker and CAI were often concomitantly prescribed. Data are presented as the mean \pm standard deviation (SD) or as percentages where appropriate. All analyses were performed using StatFlex (Atech, Osaka, Japan), with $\mathrm{P}<0.05$ considered significant.

\section{Results}

The characteristics, ophthalmological results and DED-related medications of 176 patients enrolled in this study (79 males and a mean age of 67.9 years) are detailed in Table 1. The corneal staining score was higher in groups with short BUT compared with the control group. Non-glaucoma with short BUT group was prescribed more dry eye medications compared with the other groups; more than half (56\%) of them received DED-related medications and $17-31 \%$ of patients in the other groups. Steroid eye-drops were used only for non-glaucoma groups. The distribution of seasons for BUT determination was similar in short and normal BUT groups ( $P=0.305$ for non-glaucoma groups and $P=0.118$ for glaucoma groups, Mann-Whitney test) and there was a difference between glaucoma and non-glaucoma group ( $P=0.010$, Kruskal Wallis test). 
Prostaglandin analogues provided a first-line medication for $97.2 \%(70 / 72)$ of glaucoma patients and betablockers were prescribed for 25 cases $(34.7 \%$; Table S1). The mean number of eye-drops was $1.4 \pm 0.7$ for glaucoma without short BUT group and $1.2 \pm 0.5$ for glaucoma with short BUT group $(P=0.342$, Kruskal Wallis test), and the mean frequency of instillation was $1.4 \pm 1.0$ for glaucoma without short BUT group and $1.7 \pm 1.3$ for glaucoma with short BUT group $(P=0.416)$. Monotherapy was administered for 28 cases $(77.8 \%)$ of glaucoma without short BUT group and 27 cases $(75.0 \%)$ of glaucoma with short BUT group. All of prescribed glaucoma medications contained benzalkonium chloride or other preservative; SofZiaR in TravatanzR and PolyquadR in DuotravR.

Mean IOP was significantly different between winter and summer in the short BUT groups $(\mathrm{P}<0.001$ for both glaucoma and non-glaucoma groups, paired t test), but not for the normal BUT groups $(\mathrm{P}=0.964$ for control group and $\mathrm{P}=0.489$ for glaucoma group)(Table 2 ). The mean magnitude of seasonality (winter-summer) was greater in short BUT groups than control, and it was largest in the glaucoma with short BUT. Comparison of IOP across seasons between glaucoma groups revealed significantly lower IOP in the short BUT group for all seasons except winter.

The individual range of IOP was smaller for the glaucoma groups than the non-glaucoma groups as indicated in minimum and maximum inter-individual variation across four seasons; 10 and $10 \mathrm{mmHg}$ for glaucoma groups with and without short BUT, respectively, and 17 and $20 \mathrm{mmHg}$ for non-glaucoma groups. The graphic representation of the magnitude of seasonality are shown in Figures 1 and S1. 


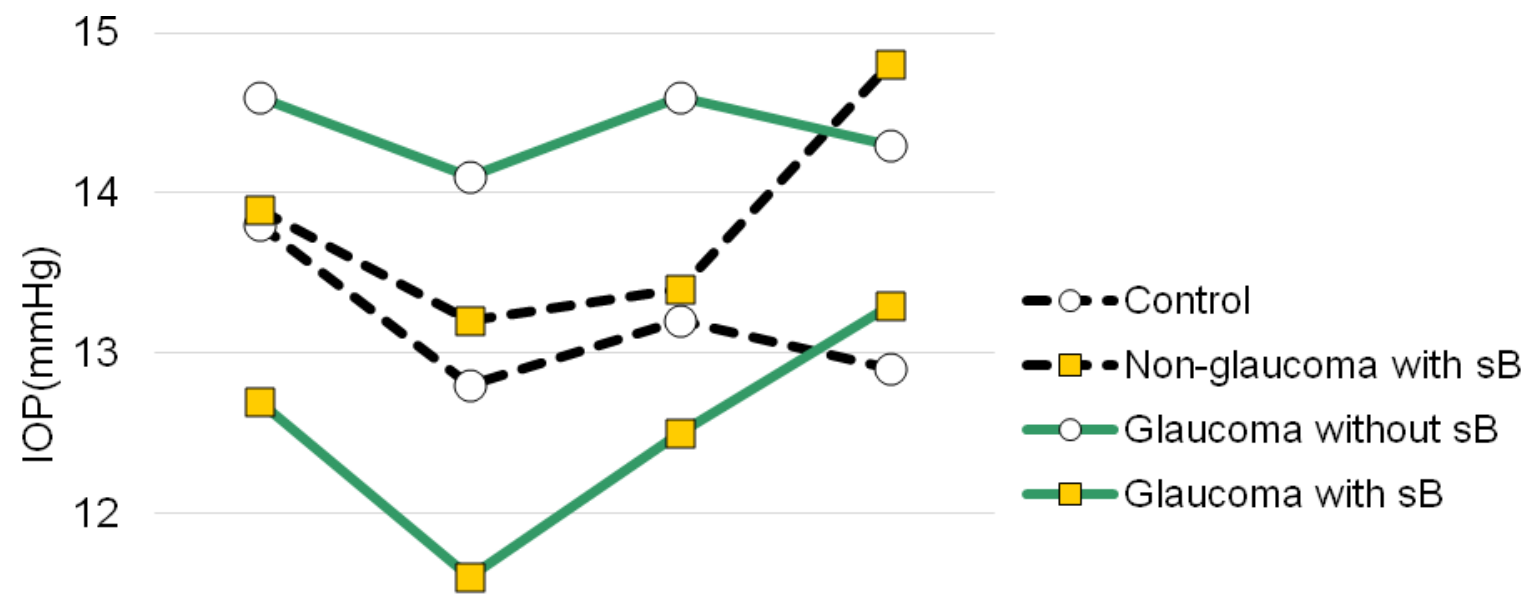

11<smiles>[GeH2]=[GeH2]</smiles><smiles>[Co]=[Co]</smiles><smiles>C1=CC=C1</smiles>

Season

Figure 1. Seasonal variation in intra-ocular pressure (IOP).

Mean IOP value in each group presenting for IOP measurements was higher in winter than in summer.

Study groups with short tear break-up time (sB) showed larger fluctuation than those groups without sB.

Table 1. Demographics and dry eye medication in each group.

\begin{tabular}{lcccc}
\hline & Control & $\begin{array}{c}\text { Non-glaucoma } \\
\text { with sB }\end{array}$ & $\begin{array}{c}\text { Glaucoma } \\
\text { without sB }\end{array}$ & $\begin{array}{c}\text { Glaucoma } \\
\text { with sB }\end{array}$ \\
\hline \# Participants & 47 & 57 & 36 & 36 \\
Age (years) & $71.5 \pm 14.1$ & $\begin{array}{c}66.5 \pm 15.3 \\
(0.144)\end{array}$ & $\begin{array}{c}64.1 \pm 13.8 \\
(0.028)\end{array}$ & $\begin{array}{c}71.1 \pm 13.6 \\
(1.000)\end{array}$ \\
& & 24.6 & 75.0 & 47.2 \\
\% of men & 44.7 & $(0.030)$ & $(0.010)$ & $(0.818)$ \\
& & $-1.41 \pm 2.85$ & $-2.77 \pm 3.64$ & $-1.96 \pm 3.40$ \\
Refractive errors (diopter) & $-0.68 \pm 2.78$ & $(1.000)$ & $(0.022)$ & $(0.191)$ \\
\# eyes with IOL (0/1/2) & $37 / 6 / 4$ & $44 / 3 / 10$ & $30 / 2 / 4$ & $24 / 4 / 8$ \\
Corneal staining score & $0.09 \pm 0.35$ & $0.37 \pm 0.61$ & $0.17 \pm 0.38$ & $0.53 \pm 0.74$ \\
& & $(0.018)$ & $(0.913)$ & $(0.002)$ \\
\hline Dry eye medication (\%) & & & & \\
$\quad$ None & 76.6 & $(0.001)$ & $(0.451)$ & $(0.464)$ \\
$\quad$ Hyaluronate & 10.6 & 21.1 & 11.1 & 19.4 \\
$\quad$ Mucin secretagogue & 14.9 & 35.1 & 5.6 & 11.1 \\
$\quad$ Steroid & 4.3 & 10.5 & 0 & 0 \\
Season of visit to determine BUT & & & & \\
\hline
\end{tabular}




\begin{tabular}{|c|c|c|c|c|}
\hline Spring & 0 & 1 & 3 & 5 \\
\hline Summer & 44 & 48 & 16 & 22 \\
\hline Fall & 2 & 1 & 6 & 4 \\
\hline Winter & 1 & 7 & 11 & 7 \\
\hline \multicolumn{5}{|l|}{ Glaucoma parameters } \\
\hline Mean deviation $(\mathrm{dB})$ & & & $-4.46 \pm 5.76$ & $\begin{array}{c}-5.61 \pm 5.14 \\
(0.386)\end{array}$ \\
\hline Disc cupping $(\%)$ & & & $73.2 \pm 16.8$ & $\begin{array}{c}75.7 \pm 17.8 \\
(0.957)\end{array}$ \\
\hline
\end{tabular}

Table 2. Mean intra-ocular pressure $(\mathrm{mmHg})$ in each season.

\begin{tabular}{|c|c|c|c|c|c|c|}
\hline & Control & $\begin{array}{l}\text { Non-glaucoma } \\
\text { with sB }\end{array}$ & $\begin{array}{r}\text { Glaucoma } \\
\text { without sB }\end{array}$ & $\begin{array}{l}\text { Glaucoma } \\
\text { with sB }\end{array}$ & $P$ value ${ }^{\mathrm{C}}$ & $P$ value ${ }^{\mathrm{D}}$ \\
\hline Spring & $13.8 \pm 3.3$ & $13.9 \pm 3.5$ & $14.6 \pm 3.3$ & $12.7 \pm 3.0$ & 0.973 & 0.021 \\
\hline Summer & $12.8 \pm 3.1$ & $13.3 \pm 3.4$ & $14.1 \pm 3.4$ & $11.6 \pm 2.9$ & 0.747 & 0.001 \\
\hline Fall & $13.2 \pm 3.1$ & $13.4 \pm 3.4$ & $14.6 \pm 3.9$ & $12.5 \pm 3.1$ & 0.844 & 0.008 \\
\hline Winter & $12.8 \pm 3.7$ & $14.8 \pm 3.4$ & $14.3 \pm 3.2$ & $13.3 \pm 3.0$ & 0.218 & 0.112 \\
\hline$P$ value (summer vs winter) & 0.964 & $<0.001$ & 0.489 & $<0.001$ & & \\
\hline $\begin{array}{l}\text { Mean magnitude of seasonality }{ }^{\mathrm{A}} \\
(\mathrm{mmHg})\end{array}$ & $0.0 \pm 3.2$ & $\begin{array}{l}1.5 \pm 2.8 \\
(0.023)\end{array}$ & $\begin{array}{l}0.2 \pm 2.1 \\
(1.000)\end{array}$ & $\begin{array}{l}1.7 \pm 2.3 \\
(0.032)\end{array}$ & 0.091 & 0.009 \\
\hline Highest seasonality ${ }^{\mathrm{B}}$ & 8 & 7 & 5 & 6 & & \\
\hline Lowest seasonality & -12 & -10 & -5 & -4 & & \\
\hline Range (highest-lowest) & 20 & 17 & 10 & 10 & & \\
\hline
\end{tabular}

${ }^{\mathrm{A}} P$ value compared with control in parentheses, using Mann-Whitney $U$ test with Bonferroni correction.

$\mathrm{A}=($ winter-summer $),{ }^{\mathrm{B}}=$ maximum value of the magnitude, ${ }^{\mathrm{C}}=$ control vs non-glaucoma with $\mathrm{sB},{ }^{\mathrm{D}}=$ glaucoma without sB vs glaucoma with sB. Abbreviations: sB, short tear break-up time.

Linear regression analysis revealed that the magnitude of seasonality in IOP was correlated with BUT and the number and frequency of medication, whereas it was not correlated with age, sex, corneal staining score, mean deviation, refractive errors, and the type of medication (Table 3). Multiple regression analysis demonstrated BUT was most strongly correlated with seasonality among three variables.

Table 3. Regression analysis of the magnitude of IOP and parameters.

\begin{tabular}{lcccc}
\hline & \multicolumn{2}{c}{ Linear regression } & \multicolumn{2}{c}{ Adjusted for age and sex } \\
& $\beta$ & $P$-value & $\beta$ & $P$-value \\
\hline Age & -0.077 & 0.519 & & \\
Sex ${ }^{\mathrm{A}}$ & 0.019 & 0.874 & & \\
Model 1: Corneal parameters & & & & \\
Tear break-up time (sec) & -0.224 & $0.003^{*}$ & -0.237 & $0.002^{*}$ \\
Corneal staining score & 0.100 & 0.403 & 0.126 & 0.107 \\
Model 2: Glaucoma-related parameters & & & & \\
\hline
\end{tabular}




\begin{tabular}{lcccc}
\hline Beta blocker & 0.084 & 0.581 & 0.067 & 0.662 \\
Carbonic anhydrase inhibitor & 0.128 & 0.399 & 0.156 & 0.320 \\
Number of medications & 0.269 & $0.022^{*}$ & 0.266 & $0.025^{*}$ \\
Frequency of medication & 0.246 & $0.037^{*}$ & 0.247 & $0.039^{*}$ \\
Refractive error (D) & 0.007 & 0.929 & -0.044 & 0.569 \\
Mean Deviation (dB) & -0.123 & 0.320 & -0.136 & 0.281 \\
Disc cupping (\%) & -0.063 & 0.617 & -0.049 & 0.712 \\
& & & & \\
Multiple regression & Non-adjusted & Adjusted for age and sex \\
Tear break-up time & -0.292 & $0.011^{*}$ & -0.356 & $0.004^{*}$ \\
Number of medications & 0.372 & 0.346 & 0.237 & 0.572 \\
Frequency of medication & -0.141 & 0.720 & -0.013 & 0.973 \\
\hline
\end{tabular}

${ }^{\mathrm{A}}$ male $=1 ;$ female $=0 .{ }^{*} P<0.05$.

Abbreviations: CAI, carbonic anhydrase inhibitor; NFL, thickness of peripapillary nerve fiber layer.

\section{Discussion}

The present results indicated the following two major findings: seasonality of IOP was greater with a short BUT compared to normal BUT, and IOP was lower with short BUT than with normal BUT. Based on the reported seasonal variation in $\mathrm{DED},[7]$ the Schirmer test value was worst in winter, with BUT and corneal staining scores the second worst in winter for DED patients. In contrast, all of the corneal signs were less severe in summer. It is notable that seasonal variation in IOP in our study was similar to that reported for corneal staining score in a DED group described previously.[7] Corneal damage induce inflammation on the ocular surface leading to IOP increases mediated by bioactive molecules including TGF beta [26,27] and prostaglandins.[28] Distress associated with uncomfortable symptoms including irritation, pain, and dryness could also raise IOP, particularly because depression is prevalent in DED patients and worsens in winter, and thus this state might result in ocular hypertension. [29-32] Such an outcome might also explain the IOP reduction noted in summer when the corneal staining scores and DED symptoms are least severe. IOP and blood pressure are positively correlated and increased in winter, $[13,14]$ and adrenergic receptor activation has been conventionally proposed as a possible regulatory system for this phenomenon.[33] IOP fluctuations are generally differentiated in short-term (diurnal) and long-term IOP fluctuations (months - years) and the seasonal 
IOP fluctuation is the latter one. Our results suggested it could be present in both glaucoma and non-glaucoma cases.

The present results indicated that IOP was lower in cases with short BUT than in those with normal BUT, and this difference was larger between glaucoma groups than between non-glaucoma groups. Taken together, lower IOP values and larger winter IOP rises were predominantly observed in glaucoma cases with short BUT. This is paradoxical since if IOP increases with worsening of DED in the cold and dry weather of winter, IOP in short BUT should be higher than in normal BUT for all seasons. A possible explanation for this seeming anomaly is cornel thinning and drug penetration facilitated by disruption of the ocular surface barrier effects in DED as previous investigations suggested IOP may be estimated for lower value in thin cornea and intra-ocular drug effects of instilled eyedrop depend on drug penetration.[12,34,35] Sleep disorder, depression, and possible decreases in melatonin secretion could also contribute to the dysregulation of IOP[36] in winter when sunshine decreases and DED patients may suffer more stress and worsened symptoms.

Our study has several limitations. First, because this is an observational study, unmeasured or residual confounding factors may remain. Nonetheless, our multivariable adjusted analyses of major possible contributory factors should attenuate potential errors. Second, there is a fundamental limitation in the lack of data for corneal thickness and blood pressure because we failed to confirm whether thinner corneal thickness in DED including cases with short BUT and seasonal variation in blood pressure were directly attributed to the seasonal variation in IOP or other relevant neuronal factors such as use of neuronal or psychiatric medications in glaucoma cases. Therefore, future longitudinal studies with systemic evaluations of neuronal aspects are necessary to gain an understanding of seasonal IOP variation in glaucoma patients with short BUT. Third, because the majority of our participants were Japanese, our data may lack generalizability. Hence, additional studies performed in patients with different ethnicities are warranted to investigate the association between seasonality and BUT. Thus, we interpreted age-/sex- adjusted and multivariable-adjusted models in addition to univariable model results with some caution. Fourth, we should have used Goldmann tonometer as a gold standard for IOP measurement, however, we believe IOP data was obtained accurately enough for our study since most examinations were performed by certified orthoptists (national licensure) and we analyzed the mean IOP of three measurements. Finally, potential selection bias and heterogeneity among intergroups might not be completely eliminated, although the distribution of baseline characteristics for age and sex were reasonable in that more women were included in the short BUT groups and more myopic subjects were included in the glaucoma groups. Also, our study examined BUT once at the time of study entry and BUT values can vary among seasons on an individual basis. Thus, BUT and IOP could be measured in each season and a mixed effect 
model applied, although the distribution of the season for BUT measurement was uniformly distributed in the present study enabling us to address this issue.

This study has several strengths. First, this study was conducted in Japan with four distinct seasons presenting various temperature and humidity fluctuations. Second, the samples were collected from multiple institutions in Japan, allowing us to conduct a case-control study including enriched ophthalmic parameters in a rigorous manner. The novelty of our current study is that we successfully capture the distinctly enhanced seasonal variation in glaucoma patients accompanied with short BUT-type DED, with being very common and severely affecting quality of life through eye disease. Additionally, the present results were demonstrated as comparable with those of a large-scale study over 4000 cases to minimize a variety of bias.[37] In a large-scale study we examined each patient once to immediately correlate IOP with DED, and the present study was carried out in a standard method with repeated examinations on the same patients to adequately analyze seasonal variation.

In conclusion, the eye care specialists should be careful of IOP in glaucoma cases with short BUT since seasonal IOP fluctuation may be greater than normal BUT cases and special attention should be paid for IOP rise in winter. BUT measurement could be recommended in glaucoma cases since any glaucoma medication may cause or aggravate DED and it could be associated with considerable IOP fluctuation.

Author Contributions: Conceptualization, M.A. and K. N.; Data curation, M.A.; Formal analysis, M.A.; Investigation, K.N. and M.A.; Methodology, M.A.; Project administration, K.T. and K.N.; Resources, M.A.; Software, M.A.; Supervision, K.T. and K.N.; Validation, K.Y., M.U, M.K., and M.A.; Visualization, M.A.; Writing - original draft, M.A.; Writing review \& editing, M.A., K.Y., M.K., M.U., K.T. and K.N.

Funding: There is no funding for this study.

Conflicts of Interest:The authors declare no conflict of interest.

Acknowledgments: We would like to thank the participants and staff of the Komoro Kosei General Hospital (Nagano, Japan), Shinseikai Toyama Hospital (Toyama, Japan), Tsukuba Central Hospital (Ibaraki, Japan), Jiyugaoka Ekimae Eye Clinic (Tokyo, Japan), Todoroki Eye Clinic (Tokyo, Japan), and Takahashi-Hisashi Eye Clinic (Akita, Japan).

\section{References}

1. Weinreb RN, Khaw PT. Primary open-angle glaucoma. Lancet. 2004; 363:1711-1720. 
2. Tsubota K, Yokoi N, Shimazaki J, Watanabe H, Dogru M, Yamada M, Kinoshita S, Kim HM, Tchah HW, Hyon JY, Yoon KC, Seo KY, Sun X, Chen W, Liang L, Li M, Liu Z; Asia Dry Eye Society. New Perspectives on Dry Eye Definition and Diagnosis: A Consensus Report by the Asia Dry Eye Society. Ocul Surf. 2017; 15:65-76.

3. Iwase A, Suzuki Y, Araie M, Yamamoto T, Abe H, Shirato S, Kuwayama Y, Mishima HK, Shimizu H, Tomita G, Inoue Y, Kitazawa Y; Tajimi Study Group, Japan Glaucoma Society. The prevalence of primary open-angle glaucoma in Japanese: the Tajimi Study. Ophthalmology. 2004;111:1641-8.

4. Uchino M, Nishiwaki Y, Michikawa T, et al. Prevalence and risk factors of dry eye disease in Japan: Koumi study. Ophthalmology. 2011;118:2361-7.

5. Ayaki M, Iwasawa A, Niwano Y. Cell viability score as an integrated indicator for cytotoxicity of benzalkonium chloride-containing antiglaucoma eyedrops. Biocontrol Sci. 2012;17:121-8.

6. Kass MA, Heuer DK, Higginbotham EJ, et al. The Ocular Hypertension Treatment Study: a randomized trial determines that topical ocular hypotensive medication delays or prevents the onset of primary open-angle glaucoma. Arch Ophthalmol. 2002;120:701-13.

7. Ayaki M, Kawashima M, Uchino M, et al. Possible association between subtypes of dry eye disease and seasonal variation. Clin Ophthalmol. 2017;11:1769-75.

8. van Setten G, Labetoulle M, Baudouin C, Rolando M. Evidence of seasonality and effects of psychometry in dry eye disease. Acta Ophthalmol. 2016;94:499-506.

9. Tesón M, López-Miguel A, Neves H, et al. Influence of climate on clinical diagnostic dry eye tests: pilot study. Optom Vis Sci. 2015;92: e284-e289.

10. Kumar N, Feuer W, Lanza NL, Galor A. Seasonal variation in dry eye. Ophthalmology. 2015;22:1727-9.

11. Gordon MO, Beiser JA, Brandt JD, et al. The Ocular Hypertension Treatment Study: baseline factors that predict the onset of primary open-angle glaucoma. Arch Ophthalmol 2002;120:714-20.

12. Brandt JD, Beiser JA, Kass MA, Gordon MO. Central corneal thickness in the Ocular Hypertension Treatment Study (OHTS). Ophthalmology 2001;108:1779-88

13. Yoshida M, Ishikawa M, Karita K, Kokaze A, Harada M, Take S, Ohno H. Association of blood pressure and body mass index with intraocular pressure in middle-aged and older Japanese residents: a cross-sectional and longitudinal study. Acta Med Okayama. 2014;68:27-34.

14. Kawase K, Tomidokoro A, Araie M, Iwase A, Yamamoto T; Tajimi Study Group; Japan Glaucoma Society. Ocular and systemic factors related to intraocular pressure in Japanese adults: the Tajimi study. Br J Ophthalmol. 2008;92:1175-9.

15. Koga T, Tanihara H. Seasonal variant of intraocular pressure in normal and glaucomatous eyes. Japan J Clin Ophthalmol. 2001;55:1519-22. In Japanese

16. Clark CV, Mapstone R. Autonomic neuropathy in ocular hypertension. Lancet. 1985;2:185-7.

17. Bengtsson B. Some factors affecting the distribution of intraocular pressures in a population. Acta Ophthalmol (Copenh) 1972;50:33-46. 
18. Blumenthal M, Blumenthal R, Peritz E, et al. Seasonal variation in intraocular pressure. Am J Ophthalmol 1970;69:608-10.

19. Qureshi IA, Xi XR, Lu HJ, et al. Effect of seasons upon intraocular pressure in healthy population of China. Korean J Ophthalmol 1996;10:29-33.

20. Qureshi IA, Xiao RX, Yang BH, et al. Seasonal and diurnal variations of ocular pressure in ocular hypertensive subjects in Pakistan. Singapore Med J 1999;40:345-8.

21. Gardiner SK, Demirel S, Gordon MO, et al. Ocular Hypertension Treatment Study Group. Seasonal changes in visual field sensitivity and intraocular pressure in the ocular hypertension treatment study. Ophthalmology 2013;120:72430.

22. Dartt DA. Neural regulation of lacrimal gland secretory processes: relevance in dry eye diseases. Prog Retin Eye Res. 2009;28:155-177.

23. Shimazakim J, Tsubota K, Kinoshita S, Ohashi Y. Definition and diagnosis of dry eye 2006. Atarashii Ganka. $2007 ; 24: 181-4$.

24. Ayaki M, Tsuneyoshi Y, Yuki K, Tsubota K, Negishi K. Latanoprost may exacerbate progression of presbyopia. PLoS One. 2019;14:e0211631.

25. Ayaki M, Kuze M, Kondo M, Tsubota K, Negishi K. Association between Retinal Nerve Fiber Layer Thickness and Eye Fatigue Biomed Res Int. 2019;2019:3014567.

26. Zheng X, De Paiva CS, Rao K, et al. Evaluation of the Transforming Growth Factor $\beta$ Activity in Normal and Dry Eye Human Tears by CCL-185 Cell Bioassay. Cornea. 2010; 29:1048-54.

27. Hill LJ, Mead B, Thomas CN, et al. TGF- $\beta$-induced IOP elevations are mediated by RhoA in the early but not the late fibrotic phase of open angle glaucoma. Mol Vis. 2018;24:712-26.

28. Yamagishi-Kimura R, Honjo M, Aihara M. Contribution of prostanoid FP receptor and prostaglandins in transient inflammatory ocular hypertension. Sci Rep. 2018;8:11098.

29. Yamamoto K, Sakamoto Y, Irie M, Ohmori S, Yoshinari M, Kaçaniku G. The relationship between IMPS-measured stress score and intraocular pressure among public school workers. J Physiol Anthropol. 2008;27:43-50.

30. Patten SB, Williams JV, Lavorato DH, et al. Seasonal variation in major depressive episode prevalence in Canada. Epidemiol Psychiatr Sci. 2017;26:169-76.

31. Labbe A, Wang YX, Jie Y, Baudouin C, Jonas JB, Xu L. Dry eye disease, dry eye symptoms and depression: the Beijing Eye Study. Br J Ophthalmol. 2013;97:1399-1403.

32. Ayaki M, Tsubota K, Kawashima M, et al. Sleep Disorders are a Prevalent and Serious Comorbidity in Dry Eye. Inv Ophth Vis Sci. 2018;59:DES143-DES150.

33. Bartels SP, Roth HO, Jumblatt MM, Neufeld AH. Pharmacological effects of topical timolol in the rabbit eye. Invest Ophthalmol Vis Sci. 1980;19:1189-97.

34. Ali NM, Hamied FM, Farhood QK. Corneal thickness in dry eyes in an Iraqi population. Clin Ophthalmol. $2017 ; 11: 435-40$ 
35. Pflugfelder SC, de Paiva CS. The Pathophysiology of Dry Eye Disease: What We Know and Future Directions for Research. Ophthalmology. 2017;124:S4-S13.

36. Lledó VE, Alkozi HA, Pintor J.Yellow filter effect on melatonin secretion in the eye: Role in IOP regulation. Curr Eye Res. 2019 Jan 14. doi: 0.1080/02713683.2019.1570276.

37. Kuze M, Ayaki M. Seasonal variation of intra-ocular pressure in glaucoma with and without dry eye. Sci Rep. (under review).

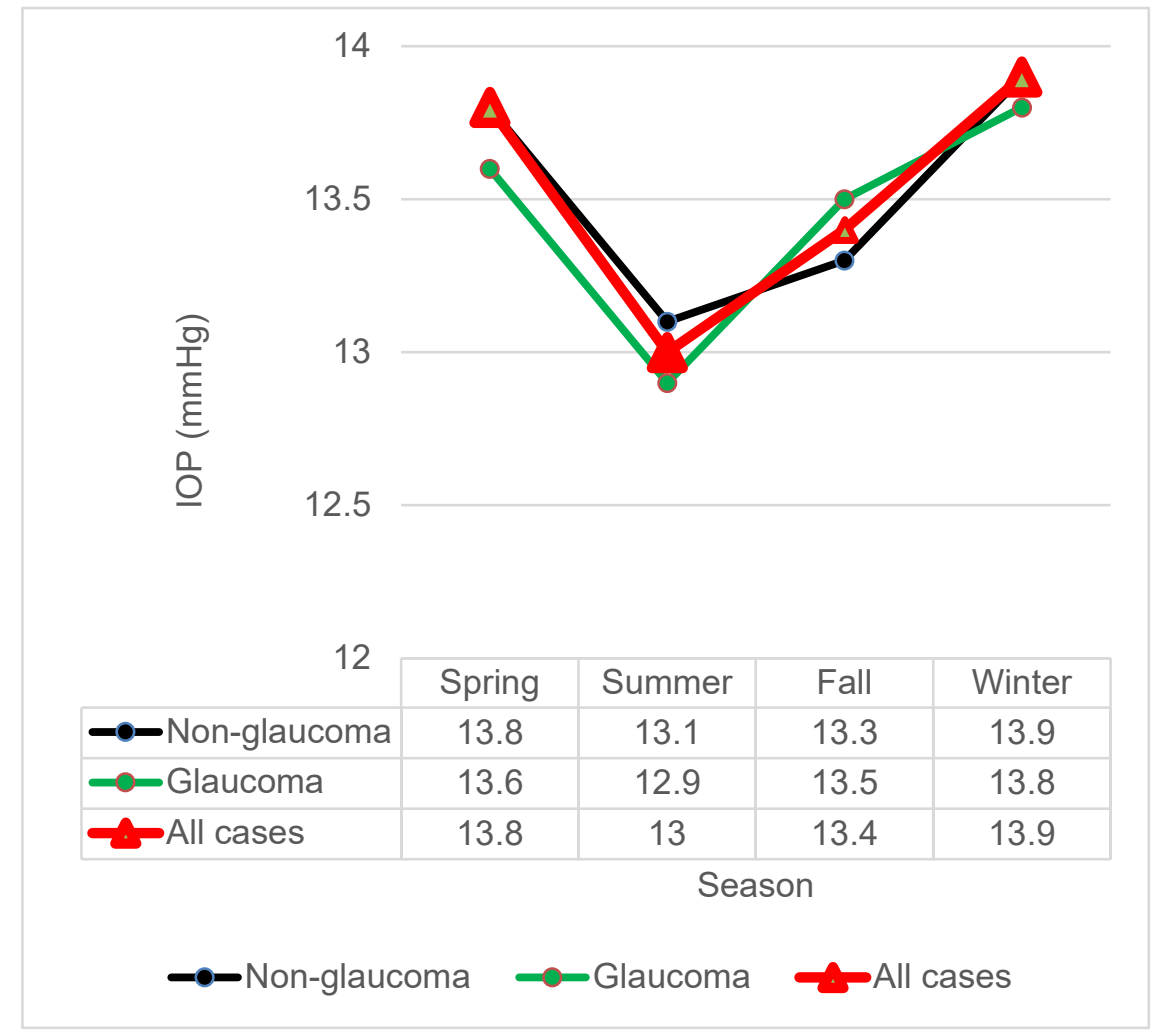

Figure S1. Intra-ocular pressure (IOP) of glaucoma and non-glaucoma groups

Table S1. Glaucoma medications in each group.

\begin{tabular}{lccc}
\hline & & Glaucoma without & Glaucoma with short \\
& & short BUT & BUT \\
\hline \# of medication & 1 & 28 & 5 \\
\hline
\end{tabular}


Mean*

Frequency of instillation

Medications

PG

Latanoprost

Tafluprost

Travoprost

Bimatoprost

Fixed combination

PG/timolol

CAI/timolol

Beta blocker

Timolol

Carteolol

CAI Dorzolamide

Brinzomide

Other $^{\mathrm{A}}$
$1.4 \pm 0.7$

$1.2 \pm 0.5$

27

4

3

3

1

$1.7 \pm 1.3$

$1.4 \pm 1.0$

20

11

3

2

6

15

1

5

3

4

$\overline{\mathrm{A}}=$ Brimonidine tartrate for three and ripasudil hydrochloride hydrate for one. Abbreviations; BUT, tear break-up time; PG, prostaglandin analogue; CAI, carbonic anhydrase inhibitor. $* P=0.342, * * P=0.416$ (Kruskal-Wallis).

All of prescribed glaucoma medications contained benzalkonium chloride or other preservative; SofZia in travoprost eyedrop and Polyquad in fixed combination with travoprost and timolol maleate. Active compounds of glaucoma medication prescribed in the present study are as follows: $0.005 \%$ latanoprost, $0.0015 \%$ tafluprost, $0.004 \%$ travoprost, $0.003 \%$ bimatoprost, fixed combination of $0.005 \%$ latanoprost and $0.5 \%$ timolol maleate, fixed combination of $0.0015 \%$ tafluprost and $0.5 \%$ timolol maleate, fixed combination with $0.004 \%$ travoprost and $0.5 \%$ timolol maleate, fixed 
combination with $0.5 \%$ timolol maleate and $1 \%$ dorzolamide hydrochloride, $1 \%$ brinzolamide, $1 \%$ dorzoramide hydrochloride, $0.1 \%$ brimonidine tartrate, $2 \%$ carteolol hydrochloride, $0.4 \%$ ripasudil hydrochloride hydrate, and $0.5 \%$ timolol maleate. 